\title{
Incidence of Bloodstream Infections in a Speciality Hospital in Kuwait: 8-Year Experience
}

\author{
T.S. Dimitrov ${ }^{\mathrm{a}} \quad$ D. Panigrahi ${ }^{\mathrm{b}} \quad$ M. Emara ${ }^{\mathrm{a}} \quad$ A. Al-Nakkas ${ }^{\mathrm{a}} \quad$ F. Awni ${ }^{\mathrm{a}}$ \\ R. Passadilla ${ }^{a}$

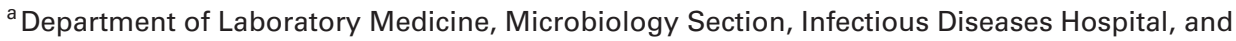 \\ ${ }^{b}$ Department of Laboratory Medical Sciences, Faculty of Allied Health Sciences, Kuwait University, Kuwait
}

\section{Key Words}

Bloodstream infections · Frequency of isolation •

Antimicrobial susceptibility

\begin{abstract}
Objectives: To determine the frequency of isolation and antibiotic-susceptibility patterns of clinically significant bacterial pathogens isolated from blood. Materials and Methods: The study was conducted over a period of 8 years (1995-2002) at Infectious Diseases Hospital (IDH), Kuwait. Demographic and clinical data were obtained from medical records. 18,535 blood cultures were analyzed. Disk diffusion method was used to perform antibiotic-susceptibility testing. Minimum inhibitory concentrations of 9 antimicrobials were determined using E-test. Double disk (potentiation) test and E-test ESBL strips were used to detect the production of extended-spectrum beta-lactamases (ESBLs). Results: Salmonella spp. and Brucella spp. were predominant blood isolates, and represented 60.6 and $30.0 \%$ of all clinically significant episodes of bloodstream infections, respectively. Among the Salmonella, Salmonella enterica serotypes typhi and paratyphi $A$ were most frequently isolated. The percentage of multidrug resistance (MDR) among them varied from 22 to $51 \%$. A high percentage (40\%) of MDR S. enterica serotypes typhi and paratyphi $A$ also showed reduced susceptibility to ceftriaxone and ciprofloxacin.
\end{abstract}

Conclusion: During the study period, Salmonella spp. and Brucella spp. were predominant blood isolates. MDR $S$. enterica serotypes typhi and paratyphi $A$, with reduced susceptibility to ceftriaxone and ciprofloxacin, are among the most frequent causes of bloodstream infections in IDH, suggesting the need to monitor their susceptibility.

Copyright $\subset 2005$ S. Karger AG, Basel

\section{Introduction}

Bacterial bloodstream infections (BIs) are a leading cause of morbidity and mortality worldwide [1]. The detection of bacteremia and septicemia remains one of the most important services provided by clinical bacteriology laboratories [2, 3]. Management of infection in many cases is based on an empirical basis, especially in immunocompromised or elderly patients, who may not present with the usual clinical signs and symptoms. It is therefore important to be able to detect bacteremia/septicemia and to identify the causative pathogens and determine antibiotic susceptibility, so that optimal patient management can be instituted as early as possible. There are several studies available on BIs in large general and university hospitals [4-6], but fewer studies have been conducted in small and specialized hospitals. Several reports dedicated on Salmonella enterica serotypes typhi, paratyphi $A$ and Brucella spp. (as predominant blood isolates in Infectious

\section{KARGER}

Fax +4161306 1234

E-Mail karger@karger.ch

www.karger.com
C 2005 S. Karger AG, Basel

$1011-7571 / 05 / 0146-0417 \$ 22.00 / 0$

Accessible online at:

www.karger.com/mpp
Dr. Ts. Dimitrov, MD, $\mathrm{PhD}$

Senior Specialist Microbiologist, Laboratory Department, IDH

PO Box 4710

13048 Safat (Kuwait)

Tel. +965 4809642, Fax +965 4871127, E-Mail dimitrov_varn90@hotmail.com 
Diseases Hospital) have been published [7-9]. However, no report is available presenting the total picture of BIs in Infectious Disease Hospital, Kuwait, hence this retrospective study was carried out to characterize the BIs to determine the frequency of bacterial isolation and their antimicrobial susceptibility pattern.

\section{Materials and Methods}

The study was carried out in the Infectious Diseases Hospital, Kuwait over a period of 8 years (1995-2002). The Infectious Diseases Hospital is a 151-bed specialized institution serving the entire population of Kuwait. The average number of admissions to the hospital is 4,000 per year. All the patients with bacteremia diagnosed by blood culture were included in the study. Data were collected from the microbiology and patient's medical records.

\section{Blood Cultures}

Blood was drawn aseptically, using Vacutainer system (Becton Dickinson) from all adult and pediatric inpatients and outpatients with suspected BIs and directly inoculated into BD Bactec Plus Aerobic/F, BD Bactec Plus Anaerobic/F and BD Bactec Peds/F bottles. The volume of blood obtained from adult was 5-10 $\mathrm{ml}$ and for the children 1-3 $\mathrm{ml}$ as previously recommended [10]. The Bactec 9120 (Becton Dickinson Diagnostic Instrument System, Sparks, Md., USA), a continuous-monitoring blood culture system, was used for further monitoring of blood cultures. The protocols of 7-day incubation for routine cultures and 21 days for fastidious microorganisms (including Brucella) were adopted.

All bottles positive in the system were gram-stained and subcultured on suitable media (blood agar, chocolate agar, MacConkey agar, Brucella blood agar, BBA). If no organisms were seen, the bottle was returned to the system to complete the testing cycle. All clinically significant bacterial isolates were identified according to standard methods [11].

\section{Antimicrobial Susceptibility Testing}

The isolated microorganisms were tested by Kirby-Bauer disk diffusion method on Mueller-Hinton agar and BBA, and the results were interpreted according to the current National Committee for Clinical Laboratory Standards (NCCLS) guidelines [12]. Antimicrobial agents (disks) were obtained from their respective manufacturers. Escherichia coli ATCC 25922, Staphylococcus aureus ATCC 29213, Pseudomonas aeruginosa ATCC 27853, Staphylococcus epidermidis ATCC12228, Streptococcus pneumoniae ATCC 6503 and Enterococcus faecalis ATCC 19212 were used as quality control strains. Minimum inhibitory concentrations (MICs) of ampicillin, amoxicillin, clavulanic acid, cephalothin, cefuroxime, ceftriaxone, piperacillin, Tazocin, gentamicin and ciprofloxacin were determined using E-test strips (AB Biodisk, Sweden) [13]. Antimicrobial susceptibility testing for Brucella isolates was performed on BBA. Since the disk diffusion method is not standardized for $\mathrm{Bru}$ cella, the results were not categorized as susceptible, intermediate or resistant. Instead, the diameter of the zone of inhibition was used as indirect indicator to measure anti-Brucella activity. Inhibition zones of $<16 \mathrm{~mm}$ and $\geq 16 \mathrm{~mm}$ were considered to represent low and good activity, respectively [9].
Detection of Extended-Spectrum Beta-Lactamase (ESBL)

Production

Evaluation of isolates for ESBL production was done by double disk (potentiation) test and confirmed by E-test ESBL strip of Cormican et al. [13].

\section{Data Interpretation}

Episode of blood infection (BI) was defined by the isolation of one or more microorganisms from blood culture together with clinical evidence of systemic infection. A new episode was recorded for the same patient if the initial pathogen had previously been eradicated from the bloodstream or if an interval of at least 1 month had elapsed without signs of infection since the earlier episode.

The significance of isolates was judged according to the identity of the microorganism, the presence of more then one blood culture set for the same microorganism, the presence of the same microorganism as that found in the blood from another normally sterile site. The strains were regarded as multidrug resistant (MDR) if they showed resistance to three or more different classes of antibiotics.

\section{Results}

The distribution of patients by age was comparable during different years of the study (mean of 36.7 years and range of $0-65$ years). Patients more than 60 years of age and pediatric age group accounted for about 8 and $9 \%$ of the total number of BIs, respectively. The male-tofemale ratio was the same for all age groups (2.5:1).

During this period, 1,129 cases of bacteremia with 830 clinically significant episodes were diagnosed (table 1). Of the 18,535 blood cultures performed, 1,702 (9.2\%) were positive and 1,129 species of microorganisms were detected. Of these, 299 (26.5\%) were judged to be contaminants or to represent transient bacteremia without clinical significance. Coagulase-negative staphylococci (CNS), diphtheroids, Propionibacterium spp., Bacillus spp., Acinetobacter spp., Pseudomonas spp., representing 1.6\% of total blood cultures, were considered contaminants. A total of $61.4 \%$ of the contaminants were CNS (S. epidermidis, S. capitis, S. hominis, S. haemolyticus and S. auricularis). The remaining blood cultures $(\mathrm{n}=830)$, yielded clinically significant pathogens. The frequency distributions of these isolates are shown in table 2. Among all bacterial isolates, Salmonella spp. (503, 44.5\%) and Brucella spp. (249, 22\%) were predominant isolates. The other members of the family Enterobacteriaceae (E. coli, Klebsiella spp., Enterobacter spp.) accounted for only $7 \%$ of the total isolates. None of these produced ESBL. During the study period, we observed only a few cases of bacteremia caused by fastidious microorganisms such as nontypable serotypes of Haemophilus influenzae $(\mathrm{n}=2)$, 
Table 1. Details of bacterial isolates from blood cultures and their frequency of isolation

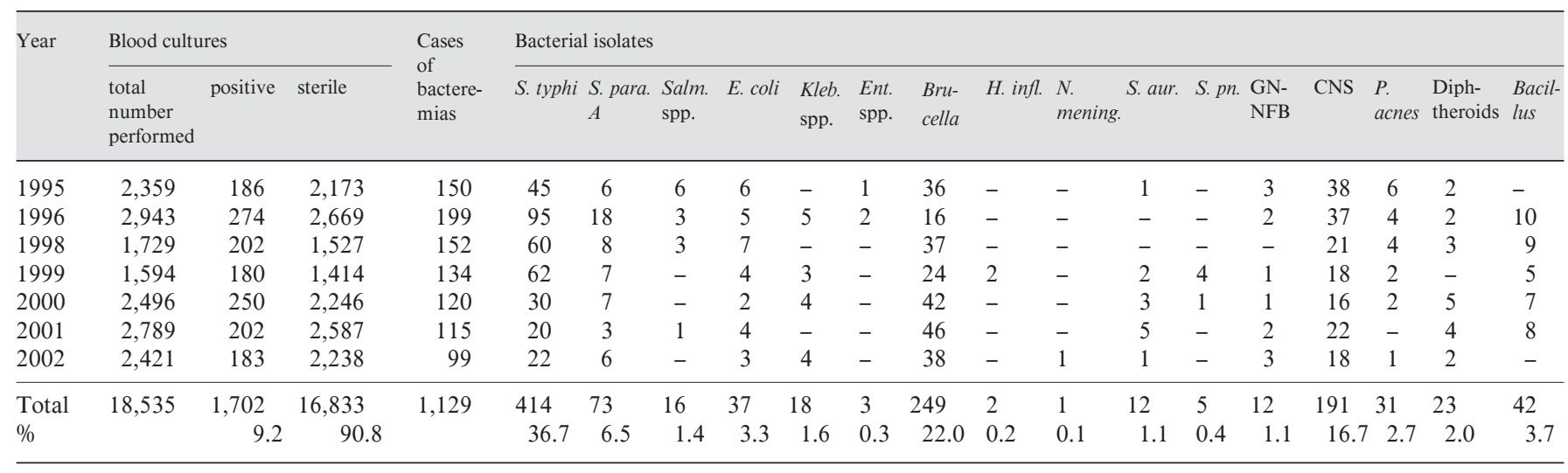

S. para. $A=S$. enterica serotype paratyphi $A ;$ Salm. = Salmonella $;$ Kleb. $=$ Klebsiella $;$ Ent. $=$ Enterobacter $;$. infl. $=H$. influenzae $; N$. mening. $=$ N. meningitidis; $S$. aur. $=S$. aureus; $S$. pn. $=$ S. pneumoniae $;$ GNNFB $=$ gram-negative nonfermenting bacteria; $P .=$ Propionibacterium acnes.

Table 2. Details of bacterial isolates from 830 clinically significant BIs (1995-2002)

\begin{tabular}{lrr}
\hline Microorganisms & Number & $\%$ \\
\hline S. enterica serotype typhi & 414 & 49.9 \\
S. enterica serotype paratyphi A & 73 & 8.8 \\
S. enterica serotypes & & \\
$\quad$ (other than typhi and paratyphi A) & 16 & 1.9 \\
E. coli & 37 & 4.5 \\
Klebsiella spp. & 18 & 2.2 \\
Enterobacter spp. & 3 & 0.4 \\
Brucella spp. & 249 & 30.0 \\
S. aureus & 12 & 1.4 \\
H. influenzae & 2 & 0.2 \\
N. meningitidis & 1 & 0.1 \\
S. pneumoniae & 5 & 0.6 \\
\hline Total species & 830 & \\
\hline
\end{tabular}

Neisseria meningitidis $(\mathrm{n}=1)$, S. pneumoniae $(\mathrm{n}=5)$. We also encountered a single episode of BI by gram-negative anaerobe, Bacteroides spp.

In less than $12 \mathrm{~h} 15 \%$ of all positive blood cultures were detected; within $24 \mathrm{~h}: 60 \%$; $48 \mathrm{~h}: 70 \% ; 72 \mathrm{~h}: 78 \%$ and within 7 days the remaining $22 \%$ were detected. At $12 \mathrm{~h}$ $45 \%$ of $S$. aureus infections and $55 \%$ of the facultative gram-negative enteric bacteria were detected. Fourteen percent of the CNS and $18 \%$ of the diphtheroids and Propionibacterium spp. were detected within $12 \mathrm{~h}$. Seventythree percent and $56 \%$ of significant and nonsignificant blood cultures, respectively, were detected during the first
Table 3. Antimicrobial susceptibility patterns (\%) of S. enterica serotype typhi recovered from blood

\begin{tabular}{|c|c|c|c|c|c|c|c|}
\hline & & Antir & icrobi & & & & \\
\hline & & $\mathrm{Am}$ & Sxt & $\mathrm{C}$ & Ctx & Cro & Cip \\
\hline $1995(n=45)$ & $\mathrm{S}$ & 38.5 & 65.9 & 65.8 & 100.0 & 100.0 & 100.0 \\
\hline & $\mathrm{R}$ & 61.5 & 34.1 & 34.2 & 0 & 0 & 0 \\
\hline $1996(n=95)$ & $\mathrm{S}$ & 69.7 & 72.4 & 69.3 & 100.0 & 100.0 & 100.0 \\
\hline & $\mathrm{R}$ & 30.3 & 27.6 & 30.7 & 0 & 0 & 0 \\
\hline $1997(n=80)$ & $\mathrm{S}$ & 47.0 & 56.3 & 48.2 & 100.0 & 100.0 & 100.0 \\
\hline & $\mathrm{R}$ & 53.0 & 43.7 & 51.8 & 0 & 0 & 0 \\
\hline $1998(\mathrm{n}=60)$ & $\mathrm{S}$ & 68.1 & 68.8 & 69.6 & 100.0 & 100.0 & 100.0 \\
\hline & $\mathrm{R}$ & 31.9 & 31.2 & 30.4 & 0 & 0 & 0 \\
\hline $1999(n=62)$ & $\mathrm{S}$ & 68.1 & 63.2 & 65.8 & 100.0 & 100.0 & 100.0 \\
\hline & $\mathrm{R}$ & 31.9 & 36.8 & 34.2 & 0 & 0 & 0 \\
\hline $2000(n=30)$ & $\mathrm{S}$ & 65.7 & 65.7 & 65.7 & 100.0 & 100.0 & 100.0 \\
\hline & $\mathrm{R}$ & 34.3 & 34.3 & 34.3 & 0 & 0 & 0 \\
\hline $2001(n=20)$ & $\mathrm{S}$ & 77.3 & 77.3 & 77.3 & 100.0 & 100.0 & 100.0 \\
\hline & $\mathrm{R}$ & 22.7 & 22.7 & 22.7 & 0 & 0 & 0 \\
\hline $2002(\mathrm{n}=22)$ & $\mathrm{S}$ & 52.0 & 52.0 & 52.0 & 100.0 & 100.0 & 100.0 \\
\hline & $\mathrm{R}$ & 48.0 & 48.0 & 48.0 & 0 & 0 & 0 \\
\hline
\end{tabular}

$\mathrm{S}=$ Sensitive; $\mathrm{R}=$ resistant; Am = ampicillin; $\mathrm{Sxt}=$ trimethoprim-sulfamethoxazole; $\mathrm{C}=$ chloramphenicol; $\mathrm{Ctx}=$ cefotaxime; Cro = ceftriaxone; $\mathrm{Cip}=$ ciprofloxacin .

$24 \mathrm{~h}$ of incubation. In all episodes of Brucella and gramnegative nonfermenting bacteria, the microorganisms were isolated only from the aerobic bottle.

The antimicrobial susceptibility results of clinically significant blood isolates are shown in tables 3 and 4 . The 
Table 4. Antimicrobial susceptibility results (\%) of E. coli and Klebsiella spp. recovered from blood cultures

\begin{tabular}{|c|c|c|c|c|c|c|c|c|c|c|}
\hline & & \multicolumn{9}{|c|}{ Antimicrobial agents } \\
\hline & & Am & Amc & $\mathrm{Cf}$ & Cxm & Cro & Pip & Tzp & $\mathrm{Gm}$ & Cip \\
\hline E. $\operatorname{coli}(\mathrm{n}=37)$ & $\mathrm{R}$ & 69.4 & 36.0 & 25.5 & 2.1 & 2.0 & 49.6 & 12.8 & 7.2 & 5.0 \\
\hline Klebsiella spp. $(\mathrm{n}=18)$ & $\mathrm{S}$ & 0 & 76.0 & 80.0 & 93.0 & 97.0 & 71.4 & 91.0 & 100.0 & 100.0 \\
\hline
\end{tabular}

$\mathrm{S}=$ Sensitive; $\mathrm{R}=$ resistant Am = ampicillin; Amc = amoxicillin/clavulanic acid; $\mathrm{Cf}=$ cephalothin; Cxm = cefuroxime; Cro = ceftriaxone; Pip = piperacillin; Tzp = Tazocin; Gm = gentamicin; Cip = ciprofloxacin .

percentage of MDR strains of Salmonella enterica serotype typhi varied from approximately $31 \%(1995,1996)$ to $44 \%$ in 1997 (table 3). During the second part of the period (1998-2001), the number of MDR $S$. typhi decreased to $23 \%$ in 2001 . Antibacterial activity of ceftriaxone and ciprofloxacin against $S$. typhi (determined by disk diffusion method) remained stable during the study period but MICs for ceftriaxone increased from 0.19 to $0.25 \mu \mathrm{g} / \mathrm{ml}$ and ciprofloxacin from 0.35 to $1.0 \mu \mathrm{g} / \mathrm{ml}$. Both E. coli and Klebsiella spp. showed a near-similar susceptibility pattern all through the study period. About $69.4,49.6,36$ and $25.5 \%$ were resistant to ampicillin, piperacillin, amoxicillin/clavulanic acid and cephalothin, respectively (table 4).

All the Brucella isolates demonstrated good susceptibility to traditional anti-Brucella drugs such as trimethoprim, tetracycline, streptomycin, gentamicin, amikacin and ciprofloxacin. Rifampicin and sulfamethoxazole had variable in vitro anti-Brucella activity with 8 and $25 \%$ of the isolates, respectively, thereby showing low potency. Not a single isolate of methicillinresistant $S$. aureus was isolated, 2 of the 5 isolates of $S$. pneumoniae expressed intermediate susceptibility to penicillin $(\mathrm{MICs}=0.75 \mu \mathrm{g} / \mathrm{ml})$.

\section{Discussion}

BIs have been reported as an important medical problem [4, 14-16], despite advances in antimicrobial therapy and supportive care. It is essential to evaluate prospectively the distribution of bacterial species isolated from blood and their susceptibility to the major antimicrobial agents and alternative drugs to adapt appropriate antibiotic therapy strategies. Ceftriaxone and ciprofloxacin are drugs of choice for the treatment of MDR enteric fever. The clinically significant bloodstream isolates of this study [Salmonella spp. (Salmonella enterica serotypes typhi and paratyphi A) and Brucella spp.] were different Enterobacteriaceae, $S$. aureus and CNS isolated from those documented in large general and university teaching hospitals [4-6, 17-20]. The probable reasons for this difference may be due to the population mix, because our hospital is a specialized hospital dealing with only infectious disease patients. Our present observation is similar to our previous reports [7-9].

In our series, $26.5 \%(299 / 1,129)$ of total isolates were judged to be contaminants or from cases of transient bacteremia without clinical significance, a finding similar to previous studies [20,21]. Of the contaminants, CNS were the predominant isolates. Several reports have documented the increasing importance of CNS as bloodstream pathogens [4, 5, 21-23], but in the present study, none of the CNS isolates met the clinical criteria to be included as cause of BIs.

The analysis of antimicrobial susceptibility patterns of $S$. enterica serotype typhi and paratyphi $A$ indicated that a considerable number of enteric fever cases in our hospital are caused by MDR strains. The percentage of MDR strains during the study period remained between 23 and $48 \%$. Panigrahi et al. [7] in an earlier study from our hospital had reported that $45 \%$ of the isolates of $S$. typhi between January 1993 and December 1994 were MDR but there were no MDR strains among S. paratyphi $A$ [8]. The percentage of MDR S. typh $i$ has decreased from 45 to $23 \%$ in 2001. Increased resistance of S. enterica serotype typhi and paratyphi $A$ to quinolones, mainly ciprofloxacin, has been widely reported $[24,25]$. In the earlier reports from our hospital no such observation was made [7, 8]. However, in the present study, we have noted that the percent- 
age of reduced susceptibility of these two Salmonella species to ciprofloxacin (MIC $>0.125 \mu \mathrm{g} / \mathrm{ml}$ ) [26] had increased up to $60 \%$ in 2002. All the Brucella isolates demonstrated susceptibility to tetracycline, gentamicin, amikacin, streptomycin and ciprofloxacin. The susceptibility pattern has remained the same as reported by us earlier [9]. In the present study other members of the family Enterobacteriaceae (E. coli, Klebsiella spp.) showed good in vitro susceptibility to imipenem, ceftriaxone, cefotaxime, ciprofloxacin and gentamicin. Resistance to aminopenicillins and first-generation cephalosporins was very high. This observation is similar to that of Decousser et al. [5].

Clinically significant gram-positive bacteremic episodes were very few in our present study. All the 12 isolates of $S$. aureus were methicillin-susceptible. This find- ing differs from that of Decousser et al. [5] where 36\% of the isolates of $S$. aureus were methicillin-resistant. In the present series, all the 12 cases were probably communityacquired infections, hence the difference. Two of the 5 isolates of S. pneumoniae (40\%) showed intermediate susceptibility to penicillin, a trend which needs to be closely monitored.

\section{Conclusion}

Salmonella enterica serotypes typhi and paratyphi $A$ were among the predominant clinically significant blood isolates in our hospital, and the number of these strains with reduced susceptibility to ciprofloxacin is increasing, thereby making the monitoring of these isolates essential.

\section{References}

1 Reimer LG, Wilson ML, Weinstein MP: Update on detection of bacteremia and fungemia. Clin Microbiol Rev 1997;10:444-465.

2 Washington JA: Blood cultures: an overview. Eur J Clin Microbiol Infect Dis 1989;8:803806.

-3 Spencer RC: Blood cultures: where do we stand? J Clin Pathol 1988;41:668-670.

-4 Haug JB, Harthug S, Kalager T, Digranes A, Solberg CO: Bloodstream infections at a Norwegian University Hospital, 1974-1979 and 1988-1989: changing etiology, clinical features, and outcome. Clin Infect Dis 1994;19: 246-256.

-5 Decousser JW, Pina P, Picot F, Delalande C, Pangon B, Courvalin P, Allouch P: Frequency of isolation and antimicrobial susceptibility of bacterial pathogens isolated from patients with bloodstream infections: a French prospective national survey. J Antimicrob Chemother 2003;51:1213-1222.

6 6 Reacher MH, Shah A, Livermore DM, Wale MC, Graham C, Johnson AP, Heine H, Monnickendam MA, Barker KF, James D, George RC: Bacteraemia and antibiotic resistance of its pathogens reported in England and Wales between 1990 and 1998: trend analysis. BMJ 2000;320:213-216.

-7 Panigrahi D, al-Aneziz AH, West PW: Plasmid-mediated multidrug resistance in Salmonella typhi in Kuwait. Trop Med Int Health 1996;1:439-442.

-8 Panigrahi D, Chugh TD, West PWJ, Dimitrov TS, Groover S, Mehta G: Antimicrobial susceptibility, phage typing and plasmid profile of Salmonella enterica serotype paratyphi $A$ strains isolated in Kuwait. Med Princ Pract 2003;12:252-255.
-9 Dimitrov TS, Panigrahi D, Emara E, Awni F, Passadilla R: Seroepidemiological and microbiological study of Brucellosis in Kuwait. Med Princ Pract 2004;13:215-219.

10 Weinstein MP: Current blood culture methods and systems: clinical concepts, technology and interpretation of results. Clin Infect Dis 1996; 23:40-46.

11 Murray PK, Baron EJ, Jurgensen JH, Pfaller MA, Yolken RH: Manual of Clinical Microbiology, ed 8. Washington, American Society for Microbiology 2003, p 1212.

12 National Committee for Clinical Laboratory Standards: Methods for Disk Susceptibility Tests for Bacteria That Grow Aerobically, ed 7. NCCLS Document M2-A7. Wayne, National Committee for Clinical Laboratory Standards, 2003.

13 Cormican MG, Marshall SA, Jones RN: Detection of extended spectrum beta-lactamase producing strains by E-test screen. Clin Microbiol 1996;34:1880-1884.

14 Reimer LG, Wilson ML, Weinstein MP: Update on detection of bacteremia and fungemia. Clin Microbiol Rev 1997;10:444-465.

15 Mycotte JM, Tayara A: Blood culture: clinical aspects and controversies. Eur J Clin Microbiol Infect Dis 2000;19:157-163.

16 Chandrasekar PH, Brown MJ: Clinical issues of blood cultures. Arch Intern Med 1994;154: 841-849.

17 Fluit AC, Jones MF, Schmitz FJ, Gupta R, Verhoef J: Antimicrobial susceptibility and frequency of occurrence of clinical blood isolates in Europe from the SENTRY antimicrobial surveillance program,1997 and 1998. Clin Infect Dis 2000;30:454-460.

18 Diekema DJ, Pfaller MA, Jones RN, Doem GV, Kugler KC, Reach ML: Trends in antimicrobial susceptibility of bacterial pathogens isolated from patients with bloodstream infections in the USA, Canada and Latin America SENTRY participants group. Int J Antimicrob Agents 2000;13:257-271.

19 Banerjee SN, Emori TG, Culver DH: Secular trends in nosocomial primary bloodstream infections in the United States, 1980-1989: National Nosocomial Infectious Surveillance System. Am J Med 1991;91(suppl 3B):86S-89S.

20 Gatell JM, Trilla A, Latorre X: Nosocomial bacteremia in a large Spanish teaching hospital: analysis of factors influencing prognosis. Rev Infect Dis 1988;10:203-210.

-21 Stillman RI, Wenzel RP, Donowitz LC: Emergence of CNS as major nosocomial bloodstream pathogens. Infect Control 1987;8:108112.

22 Mirrett S, Weinstein MP, Reimer LG, Wilson ML, Reller LB: Interpretation of coagulasenegative staphylococci in blood cultures: does the number of positive bottles help? (abstract C69). Program and Abstracts of the 93rd General Meeting of the American Society for Microbiology, Atlanta, 1993, p 458.

23 Peacock SJ, Bewler ICJW, Crook DWM: Positive predictive value of blood cultures growing coagulase-negative staphylococci (letter). Lancet 1995;346:191-192.

- 24 Chandel DS, Chaudhry R, Dhawan B, Pandey A, Dey AB: Drug-resistant Salmonella enterica serotype paratyphi $A$ in India. Emerg Infect Dis 2000;6:420-421.

25 Threlfall EJ, Ward LR: Decreased susceptibility to ciprofloxacin in Salmonella enterica serotype typhi, United Kingdom. Emerg Infect Dis 2001;7:448-450.

26 Poutanen SM, Low DE: Is it time to change fluoroquinolone MIC breakpoints for Salmonella spp.? Clin Microbiol Newsl 2003;25:97102. 